\title{
Some deviation inequalities
}

\author{
by Bernard Maurey
}

September 1990

\begin{abstract}
We introduce a concentration property for probability measures on $R^{n}$, which we call Property $(\tau)$; we show that this property has an interesting stability under products and contractions (Lemmas 1, 2, 3). Using property $(\tau)$, we give a short proof for a recent deviation inequality due to Talagrand. In a third section, we also recover known concentration results for Gaussian measures using our approach.
\end{abstract}

Introduction. Very roughly speaking, a concentration of measure phenomenon for a probability measure $\mu$ means that, given any measurable subset $A$ such that $\mu(A) \geq \frac{1}{2}$, the enlargements of $A$, in a sense to be made precise, almost have measure 1. One important example of this situation is a consequence of Paul Lévy's isoperimetric inequality for the unit sphere $S_{n}$ of $R^{n+1}$. This consequence is the following: Let $\mu_{n}$ denote the normalized rotation invariant measure on $S_{n}$; for every measurable subset $A$ of $S_{n}$ such that $\mu_{n}(A) \geq \frac{1}{2}$ we have

$$
\mu_{n}\left\{x \in S_{n} ; x \notin A_{\varepsilon}\right\} \leq \sqrt{\frac{\pi}{8}} e^{-n \varepsilon^{2} / 2}
$$

where $A_{\varepsilon}$ denotes the subset of $S_{n}$ of all points whose geodesic distance to $A$ is less than $\varepsilon$ (see [8]). This result is crucial for the proof of Dvoretzky's theorem (about almost spherical sections of convex bodies) as given by V. Milman [7] (see also Figiel, Lindenstrauss and Milman [4]). Using Lévy's result, Borell [1] was able to prove an analogous isoperimetric result for the Gaussian measure on $R^{n}$ (see also Ehrhard [3]). As it is the case for the sphere, Borell's isoperimetric result implies a Gaussian concentration of measure principle. Later, Pisier and the author gave a very simple proof for the Gaussian concentration principle which is needed for the proof of Dvoretzky's theorem (see [9], [8] and [10]). Recently, Talagrand proved [12] a concentration principle for measures on $R^{n}$ with exponential densities which is stronger than the Gaussian one (Corollary 1 below; this Corollary appears in [12] as a consequence of a more precise isoperimetric inequality which does not follow from our proof). We give here a proof for Talagrand's concentration result, using a property which we call Property $(\tau)$. This property is defined in section I, and general stability results about it are stated (Lemmas 1, 2, 3); we also explain in section I why property $(\tau)$ is related to concentration (Lemma 4). In section II, we show how to get Talagrand's inequality using property $(\tau)$. In section III we recover the Gaussian case along the same lines (Corollary 2). In a last section, we study a variant of property $(\tau)$, the convex property $(\tau)$; this variant is related to an other deviation inequality due to Talagrand [13] (see Corollary 5).

\section{Property $(\tau)$}

Let $f$ and $g$ be two measurable functions on $R^{n}$; we denote by $f \square g$ the inf-convolution of $f$ and $g$,

$$
(f \square g)(x)=\inf \left\{f(x-y)+g(y) ; y \in R^{n}\right\} .
$$

If $\mu$ is a probability measure on $R^{n}$ and $w$ a positive measurable function on $R^{n}$, we say that the couple $(\mu, w)$ satisfies the Property $(\tau)$ if for every bounded measurable function $\varphi$ on $R^{n}$ we have

$$
\left(\int e^{\varphi \mathbf{\square} w} d \mu\right)\left(\int e^{-\varphi} d \mu\right) \leq 1
$$

If we adopt the convention $+\infty .0 \leq 1$, we see easily that the above inequality extends to all $\bar{R}$-valued measurable functions $\varphi$.

The definition of property $(\tau)$ was motivated by Talagrand's isoperimetric inequality for the cube, as the careful reader of [13] will notice. 
Lemma 1. If $\left(\mu_{i}, w_{i}\right)$ satisfies $(\tau)$ on $R^{n_{i}}$ for $i=1,2$, then $\left(\mu_{1} \otimes \mu_{2}, w\right)$ satisfies $(\tau)$ on $R^{n_{1}} \times R^{n_{2}}$, where

$$
w\left(x_{1}, x_{2}\right)=w_{1}\left(x_{1}\right)+w_{2}\left(x_{2}\right) .
$$

Proof: Consider $\varphi^{y}(x)=\varphi(x, y)$ and apply $(\tau)$ to $\psi(y)=\log \left(\int e^{\varphi^{y} \mathbf{D} w_{1}} d \mu_{1}\right)$.

Lemma 2. If $\left(\mu_{i}, w_{i}\right)$ satisfies $(\tau)$ on $R^{n}$ for $i=1,2$, then $\left(\mu_{1} * \mu_{2}, w_{1} \mathbf{\square} w_{2}\right)$ satisfies $(\tau)$ on $R^{n}$.

Lemma 3. Let $\left(\mu_{1}, w_{1}\right)$ satisfy $(\tau)$ on $R^{n_{1}}$. Let $w_{2}$ be a positive measurable function on $R^{n_{2}}$ and $F$ a mapping from $R^{n_{1}}$ to $R^{n_{2}}$ such that $w_{2}(F x-F y) \leq w_{1}(x-y)$ for every pair $x, y$. Let $\mu_{2}$ be the image probability measure on $R^{n_{2}}$ defined by $\mu_{2}=F\left(\mu_{1}\right)$. Then $\left(\mu_{2}, w_{2}\right)$ satisfies $(\tau)$.

Proof: $(\varphi \circ F) \square w_{1} \geq\left(\varphi \square w_{2}\right) \circ F$.

Lipschitz maps like the above $F$ were used by Pisier [9] in a slightly different context. Following him, we will be able in section III to pass from the Gaussian case to the uniform probability measure on $[0,1]^{n}$ (Corollary 4).

We have perhaps to explain why property $(\tau)$ for a couple $(\mu, w)$ is a concentration of measure property.

Lemma 4. Assume that $(\mu, w)$ satisfies $(\tau)$ on $R^{n}$. For every measurable subset $A$ of $R^{n}$ and every positive real number $t$, we have

$$
\mu\{x \notin A+\{w<t\}\} \leq(\mu(A))^{-1} e^{-t} .
$$

Proof: Let $A$ be a measurable subset of $R^{n}$ and denote by $\varphi_{A}$ the function equal to 0 on $A$ and $+\infty$ outside. We observe that $\left(\varphi_{A} \square w\right)(x) \geq t$ when $x \notin A+\{w<t\}=\{a+y ; a \in A, w(y)<t\}$. Property $(\tau)$ implies that $\int e^{\varphi_{A} \square w} d \mu \leq(\mu(A))^{-1}$ and we conclude with Tschebycheff's inequality.

\section{Talagrand's deviation inequality}

Let us define a function $W$ on $R$ by

$$
W(t)=\frac{1}{18} t^{2} \quad \text { for } \quad|t| \leq 2, \quad \frac{2}{9}(|t|-1) \quad \text { otherwise }
$$

and let $\mu_{e}$ be the probability measure on $R$ with density $1_{(0, \infty)}(x) e^{-x}$.

Proposition. The couple $\left(\mu_{e}, W\right)$ satisfies $(\tau)$.

It follows from Lemma 2 that $(\xi, U)$ also satisfies $(\tau)$, where $\xi$ is the convolution of $\mu_{e}$ and its symmetric image on $(-\infty, 0)$, and $U=W \square W$. It is easy to see that $\xi$ has density $\frac{1}{2} e^{-|x|}$ on $R$, and that

$$
U(t)=2 W(t / 2)=\frac{1}{36} t^{2} \quad \text { for } \quad|t| \leq 4, \quad \frac{2}{9}(|t|-2) \quad \text { otherwise. }
$$

We deduce now from Lemma 1 that the couple $\left(\xi_{n}, U_{n}\right)$ satisfies $(\tau)$ on $R^{n}$ for every $n$, where $\xi_{n}$ is the product of $n$ copies of $\xi$ and $U_{n}(x)=\sum_{i=1}^{n} U\left(x_{i}\right)$. The idea of working with functions like $W$ or $U_{n}$ comes from Talagrand [12].

Theorem 1. The couple $\left(\xi_{n}, U_{n}\right)$ satisfies $(\tau)$ for every integer $n$. In particular, for every measurable subset $A$ of $R^{n}$ we have if we set $\rho_{A}=\varphi_{A} \square U_{n}$

$$
\int e^{\rho_{A}} d \xi_{n} \leq\left(\xi_{n}(A)\right)^{-1}
$$

Corollary 1 (Talagrand). For every $t>0$,

$$
\xi_{n}\left\{x ; x \notin A+6 \sqrt{t} B_{2}+9 t B_{1}\right\} \leq\left(\xi_{n}(A)\right)^{-1} e^{-t} .
$$

where $B_{2}$ and $B_{1}$ are respectively the usual $\ell_{2}^{n}$ and $\ell_{1}^{n}$ balls.

Proof: According to Lemma 4, we need only show that

$$
\left\{U_{n}<t\right\} \subset 6 \sqrt{t} B_{2}+9 t B_{1} .
$$

Assume $U_{n}(x)<t$, and define $y$ and $z$ in the following way: $y_{i}=x_{i}$ if $\left|x_{i}\right| \leq 4, y_{i}=0$ otherwise; $z_{i}=x_{i}$ if $\left|x_{i}\right|>4, z_{i}=0$ otherwise. Then $x=y+z$ and it is easy to check that $\|y\|_{2} \leq 6 \sqrt{t},\|z\|_{1} \leq 9 t$. 
We present now the proof of the above Proposition. Let $\varphi$ be a bounded measurable function on $(0,+\infty)$, and let $\psi$ denote the function $\varphi \square W$.

Let $I_{0}=\int_{0}^{\infty} e^{-\varphi(x)-x} d x$ and $I_{1}=\int_{0}^{\infty} e^{\psi(y)-y} d y$. For $t \in(0,1)$, we define $x(t)$ and $y(t)$ by the relations

$$
\int_{0}^{x(t)} e^{-\varphi(x)-x} d x=t I_{0}, \int_{0}^{y(t)} e^{\psi(y)-y} d y=t I_{1}
$$

We obtain by differentiation

$$
x^{\prime}(t)=I_{0} e^{\varphi(x(t))+x(t)}, y^{\prime}(t)=I_{1} e^{-\psi(y(t))+y(t)} .
$$

Taking into account the fact that $\psi(y(t)) \leq \varphi(x(t))+W(x(t)-y(t))$, we obtain

$$
y^{\prime}(t) \geq I_{1} e^{-\varphi(x(t))-W(x(t)-y(t))+y(t)} .
$$

Let now $z(t)=\frac{1}{2}(x(t)+y(t))-W(x(t)-y(t))$. We have

$$
z^{\prime}(t)=\left(\frac{1}{2}-W^{\prime}(x(t)-y(t))\right) x^{\prime}(t)+\left(\frac{1}{2}+W^{\prime}(x(t)-y(t))\right) y^{\prime}(t) .
$$

If we use the fact that $\left|W^{\prime}\right| \leq 1 / 2$ on $R$, we get (writing $x$ and $y$ for $x(t)$ and $y(t)$ for simplicity, and using the inequality $\frac{1}{2}(u a+v / a) \geq \sqrt{u v}$ with $\left.a=e^{\varphi(x)}\right)$

$$
\begin{gathered}
z^{\prime}(t) \geq\left(1-2 W^{\prime}(x-y)\right) I_{0} e^{x} \frac{e^{\varphi(x)}}{2}+\left(1+2 W^{\prime}(x-y)\right) I_{1} e^{-W(x-y)+y} \frac{e^{-\varphi(x)}}{2} \\
\geq \sqrt{1-4 W^{\prime}(x-y)^{2}} \sqrt{I_{0} I_{1}} e^{\frac{1}{2}(x+y)-\frac{1}{2} W(x-y)} \\
=\sqrt{I_{0} I_{1}} e^{z(t)} \sqrt{1-4 W^{\prime}(x-y)^{2}} e^{\frac{1}{2} W(x-y)}
\end{gathered}
$$

We claim that for every $s$

$$
\left(1-4 W^{\prime}(s)^{2}\right) e^{W(s)} \geq 1
$$

It will then follow that $e^{-z(t)} z^{\prime}(t) \geq \sqrt{I_{0} I_{1}}$, which yields after integrating between 0 and 1

$$
1 \geq \sqrt{I_{0} I_{1}}
$$

and this is our Proposition.

Proof of the claim: We only consider $s \geq 0$ since $W$ is even. For $s \geq 2, W^{\prime}$ is constant and $W$ increasing, so it is enough to check the case $0 \leq s \leq 2$; this reduces to

$$
e^{-u / 18} \leq 1-4 u / 81 \text { for } u \in(0,4)
$$

which is proved using elementary calculus.

\section{The Gaussian case}

Let $\gamma$ be the standard Gaussian probability measure on $R$, with density $\frac{1}{\sqrt{2 \pi}} e^{-x^{2} / 2}$, and $\gamma_{n}$ the product of $n$ copies of $\gamma$. Throughout this section, the norm will be the Euclidean norm on $R^{n}$.

Theorem 2. The couple $\left(\gamma_{n}, \frac{1}{4}\|x\|^{2}\right)$ satisfies $(\tau)$ for every integer $n$.

Proof: We check first that $\left(\gamma, x^{2} / 4\right)$ has property $(\tau)$ on $R$; the proof is similar to the proof of the Proposition, but simpler: $x(t)$ and $y(t)$ are defined in a similar fashion, and $z(t)$ is simply equal to $\frac{1}{2}(x(t)+y(t))$. It follows from Lemma 1 that $\left(\gamma_{n}, \frac{1}{4}\|x\|^{2}\right)$ has property $(\tau)$ for every integer $n$.

We can also give a direct proof using the functional Brunn-Minkowski inequality due to Prekopa and Leindler [11], [6] (see also [10]): If $f, g, h$ are bounded below measurable functions on $R^{n}$ such that for all $x$ and $u$ we have $\frac{1}{2}(f(x+u)+g(x-u)) \geq h(x)$, then

$$
\left(\int e^{-f(x)} d x\right)\left(\int e^{-g(x)} d x\right) \leq\left(\int e^{-h(x)} d x\right)^{2} .
$$

We apply this inequality to $f(x)=\varphi(x)+\frac{1}{2}\|x\|^{2}, g(y)=-\psi(y)+\frac{1}{2}\|y\|^{2}$ and $h(z)=\frac{1}{2}\|z\|^{2}$, where we have set $\psi=\varphi \square w, w(y)=\frac{1}{4}\|y\|^{2}$. 
Remark 1. The second proof of Theorem 2 only uses the uniform convexity properties of $-\log f$, where $f$ is the density of $\mu$.

Remark 2. As pointed out by Talagrand, the Gaussian concentration result is a consequence of Corollary 1 , using a suitable map that transforms $\xi_{n}$ into $\gamma_{n}$. More precisely, Lemma 3 and Theorem 1 imply that for some $a>0$, the couple $\left(\gamma_{n}, a\|x\|^{2}\right)$ satisfies $(\tau)$ for every $n$. However, the proof of Theorem 2 gives a better constant $a$ and is simpler.

We will show now that Theorem 2 allows to recover the main conclusion of the Gaussian concentration result of $[9]$.

Corollary 2. Let $\varphi$ be a 1-Lipschitz function on $R^{n}$, and $X, Y$ two independent n-dimensional Gaussian vectors with distribution equal to $\gamma_{n}$. For every real number $\lambda$ we have

$$
E e^{\frac{\lambda}{\sqrt{2}}(\varphi(X)-\varphi(Y))} \leq e^{\lambda^{2} / 2} .
$$

Remark 3. This inequality is optimal since there is equality when $\varphi$ is a norm-one linear functional.

Proof: Let $\psi=\frac{\lambda \varphi}{\sqrt{2}} \mathbf{\square} w$, where $\varphi$ is 1-Lipschitz on $R^{n}, w(y)=\frac{1}{4}\|y\|^{2}$ and $\lambda>0$. It is enough to apply $(\tau)$ and notice that $\psi(x) \geq \frac{\lambda \varphi}{\sqrt{2}}(x)-\lambda^{2} / 2$. Let $y$ be such that

$$
\psi(x)=\frac{\lambda \varphi}{\sqrt{2}}(y)+\frac{1}{4}\|x-y\|^{2} .
$$

Then

$$
\psi(x) \geq \frac{\lambda \varphi}{\sqrt{2}}(x)-\frac{\lambda}{\sqrt{2}}\|x-y\|+\frac{1}{4}\|x-y\|^{2} \geq \frac{\lambda \varphi}{\sqrt{2}}(x)+\min \left\{\frac{1}{4} u^{2}-\frac{\lambda}{\sqrt{2}} u ; u \in R\right\}=\frac{\lambda \varphi}{\sqrt{2}}(x)-\lambda^{2} / 2 .
$$

The first part of the next Corollary is known (it is a Poincaré-type inequality due to Chen [2]).

Corollary 3. If $\varphi$ is a Lipschitz function on $R^{n}$, we have

$$
\frac{1}{2} \int(\varphi(x)-\varphi(y))^{2} d \gamma_{n}(x) d \gamma_{n}(y) \leq \int\|\nabla \varphi\|^{2} d \gamma_{n} .
$$

More generally, this result holds for every probability measure $\mu$ on $R^{n}$ such that $(\mu, w)$ satisfies $(\tau)$ for a function $w$ convex and greater than $\frac{1}{4}\|x\|^{2}$ in a neighborhood of 0 .

Proof: Let $u$ be a convex function such that $u \leq w$ and $u(x)=\frac{1}{4}\|x\|^{2}$ in a neighborhood of 0 ; assume that $\varphi$ is a compactly supported $C^{1}$-function. For $t>0$ consider $\varphi_{t}=t \varphi$ and $\psi_{t}=\varphi_{t} \square u$. One can check that

$$
\lim _{t \rightarrow 0} \frac{\psi_{t}(x)-\varphi_{t}(x)}{t^{2}}=-\|\nabla \varphi(x)\|^{2}
$$

and the result follows easily from the property $(\tau)$ of $(\mu, u)$ applied to $\varphi_{t}$, when $t \rightarrow 0$.

Remark 4. If $(\mu, w)$ satisfies $(\tau)$ on $R$, where $\mu$ is such that $\frac{1}{2} \int(x-y)^{2} d \mu(x) d \mu(y)>1$, we can apply Corollary 3 to $\varphi(x)=x$ to conclude that $\left\{x ; w(x) \geq \frac{1}{4} x^{2}\right\}$ is not a neighborhood of 0 . One can also show that if $(\mu, w)$ satisfies $(\tau)$ on $R$, with $\mu$ symmetric and $\int x^{2} d \mu(x)=1$, then $\left(\gamma, \frac{1}{2} w^{\prime \prime}(0) t^{2}\right)$ also satisfies $(\tau)$. This shows the necessity of a subquadratic behavior at 0 for the function $w$.

Corollary 4. Let $\lambda_{n}$ denote the uniform probability measure on $[0,1]^{n}$. There exists $a>0$ such that $\left(\lambda_{n}, a\|x\|^{2}\right)$ satisfies $(\tau)$ for every integer $n$ (one can take $a=\pi / 2$ ).

Proof: Using Lemma 3, this follows from Theorem 2, exactly like in Pisier [9]. 


\section{Convex property $(\tau)$}

Assume that $w$ is a convex function on some topological vector space $X$ and that $\mu$ is a probability measure on $X$. We say that the couple $(\mu, w)$ satisfies the convex property $(\tau)$ provided

$$
\left(\int e^{\varphi \square w} d \mu\right)\left(\int e^{-\varphi} d \mu\right) \leq 1
$$

for every convex measurable function $\varphi$ on $X$.

Lemma 5. If $\left(\mu_{i}, w_{i}\right)$ satisfies the convex property $(\tau)$ on $X_{i}$ for $i=1,2$, then $\left(\mu_{1} \otimes \mu_{2}, w\right)$ satisfies the convex property $(\tau)$ on $X_{1} \times X_{2}$, with

$$
w\left(x_{1}, x_{2}\right)=w_{1}\left(x_{1}\right)+w_{2}\left(x_{2}\right) .
$$

Proof: As in the proof of Lemma 1 we consider $\varphi^{y}(x)=\varphi(x, y)$ for a convex function $\varphi$ on $X_{1} \times X_{2}$; we can apply the convex property $(\tau)$ to $\psi(y)=\log \left(\int e^{\varphi^{y} \square w_{1}} d \mu_{1}\right)$ if we observe that $\psi$ is a convex function.

We shall say that $\mu$ has diameter $\leq 1$ as a short way to express that $\mu$ is supported by a set of diameter $\leq 1$. The following Theorem is the equivalent in our language of a result of Talagrand [13] and its generalization by Johnson and Schechtman [5].

Theorem 3. Let $\left(X_{i}\right)$ be a family of normed spaces; for each $i$, let $\mu_{i}$ be a probability measure with diameter $\leq 1$ on $X_{i}$, and $w_{i}(x)=\frac{1}{4}\|x\|^{2}$ for $x \in X_{i}$. If $\mu$ is the product of the family $\left(\mu_{i}\right)$, then $(\mu, w)$ satisfies the convex property $(\tau)$, with $w(x)=\sum_{i} w_{i}\left(x_{i}\right)$.

Proof: According to Lemma 5, we only need to prove the result for a single probability measure $\mu$ with diameter $\leq 1$ on a normed space $X$. Let $A$ be a set of diameter $\leq 1$ that supports $\mu$, and let $\varphi$ be a convex function on $X$; assume without loss of generality that $\inf \varphi(A)=0$. Define $w(x)=\frac{1}{4}\|x\|^{2}$ and $\psi=\varphi \square w$. Let $x \in A, \varepsilon>0$ and $a \in A$ such that $\varphi(a) \leq \varepsilon$. We have, if $y=(1-\theta) x+\theta a$ and $0 \leq \theta \leq 1$

$$
\psi(x) \leq \varphi(y)+\frac{1}{4}\|x-y\|^{2} \leq(1-\theta) \varphi(x)+\theta \varepsilon+\frac{1}{4} \theta^{2} .
$$

Choosing an optimal $\theta$, we deduce from the above that $\psi(x) \leq k(\varphi(x))$ where $k(u)$ is equal to $u-u^{2}$ if $0 \leq u \leq \frac{1}{2}$, and to $\frac{1}{4}$ if $u \geq \frac{1}{2}$. We claim now that $e^{k(u)} \leq 2-e^{-u}$. It follows that

$$
\int e^{\psi} d \mu \leq 2-\int e^{-\varphi} d \mu \leq\left(\int e^{-\varphi} d \mu\right)^{-1}
$$

and this finishes the proof (the preceding computation was inspired by [5]).

Proof of the claim: For $0 \leq u \leq \frac{1}{2}$, we write

$$
\frac{1}{2}\left(e^{u-u^{2}}+e^{-u}\right)=e^{-u^{2} / 2} \cosh \left(u-u^{2} / 2\right) \leq e^{-u^{2} / 2} \cosh (u) \leq 1 .
$$

Remark 5. In the case of the probability $\beta$ on $[0,1]$ that gives measure $\frac{1}{2}$ to $\{0\}$ and $\{1\}$, it is easy to improve the function $w$ from $\frac{1}{4} x^{2}$ to $\frac{1}{2} x^{2}$.

Corollary 5 [13], [5]. Let $A$ be a measurable subset of $[0,1]^{n}$ and $B$ its convex hull. For every product probability measure $\mu$ on $[0,1]^{n}$ we have

$$
\int e^{\frac{1}{4} d_{B}^{2}} d \mu \leq(\mu(A))^{-1}
$$

where $d_{B}$ denotes the Euclidean distance to the set $B$. 


\section{References.}

[1] C. Borell, The Brunn-Minkowski inequality in Gauss space, Inventiones Math. 30 (1975), $205-216$.

[2] L. Chen, An inequality for the multivariate normal distribution, J. Multivariate Anal. 12 (1982) $306-315$.

[3] A. Ehrhard, Symétrisation dans l'espace de Gauss, Math. Scand. 53 (1983) 281-301.

[4] T. Figiel, J. Lindenstrauss, V. Milman, The dimension of almost spherical sections of convex bodies, Acta Math. 139 (1977) 53-94.

[5] W. Johnson, G. Schechtman, Remarks on Talagrand's deviation inequality for Rademacher functions, Texas Functional Analysis Seminar 1988-89.

[6] L. Leindler, On a certain converse of Hölder's inequality, Acta Sci. Math. 33 (1972) 217-223.

[7] V. Milman, A new proof of the theorem of A. Dvoretzky on sections of convex bodies, Func. Anal. Appl. 5 (1971) 28-37.

[8] V. Milman, G. Schechtman, Asymptotic theory of finite dimensional normed spaces, Springer Lecture Notes in Math. 1200 (1986).

[9] G. Pisier, Probabilistic methods in the geometry of Banach spaces, CIME Varenna 1985, Springer Lecture Notes in Math. 1206, 167-241.

[10] G. Pisier, Volume of convex bodies and Banach spaces geometry, Cambridge University Press.

[11] A. Prekopa, On logarithmically concave measures and functions, Acta Sci. Math. 34 (1973) 335-343.

[12] M. Talagrand, to appear.

[13] M. Talagrand, An isoperimetric theorem on the cube and the Khintchine-Kahane inequalities, Proc. Amer. Math. Soc. 104 (1988) 905-909.

Université Paris 7, U.F.R. de Mathématiques 